\title{
Title: COVID-19: Precautions with children in home mechanical ventilation.
}

\author{
Luis Vasconcello-Castillo ${ }^{1}$, Rodrigo Torres Castro ${ }^{2}$, Roberto Vera Uribe ${ }^{1}$, and Paiva \\ Rebeca $^{3}$ \\ ${ }^{1}$ Universidad de Chile \\ ${ }^{2}$ University of Chile \\ ${ }^{3}$ Gobierno de Chile Ministerio de Salud
}

May 26, 2020

Title: COVID-19: Precautions with children in home mechanical ventilation.

Authors: Luis Vasconcello-Castillo ${ }^{1}$, Rodrigo Torres-Castro $\mathrm{MSc}^{1}$, Roberto Vera-Uribe MSc ${ }^{1}$, Rebeca Paiva $\mathrm{MD}^{2}$

${ }^{1}$ Departamento de Kinesiología, Facultad de Medicina, Universidad de Chile, Santiago, Chile

${ }^{2}$ Ministerio de Salud, Programa de Asistencia Ventilatoria No Invasiva Domiciliaria, Santiago, Chile

Financing

Not funding

\section{Correspondence}

Luis Vasconcello-Castillo, Departamento de Kinesiología, Universidad de Chile, Independencia 1027, Santiago, Chile. Phone number: (562) 29786513. E-mail: l.vasconcello.c@gmail.com

Key words: COVID-19; Home mechanical ventilation; children; telemedicine

Abbreviated title: COVID-19 in home mechanical ventilation

To the editor,

COVID-19 has become a global public health problem. The virus mainly affects the respiratory system, and its most common symptoms are cough, dyspnea, fever, headache, and myalgia. ${ }^{1}$ It is also characterized by a rapidly evolving severe respiratory condition, with approximately $20 \%$ of those infected requiring hospitalization and $6 \%$ in critical care and needing invasive ventilatory assistance. ${ }^{1}$

Although this disease has been shown to be less severe and less prevalent in children, ${ }^{1}$ a particular group of children are considered a risk group for developing a severe COVID-19 infection, namely, those who use home mechanical ventilation (HMV). This group is composed of various groups of pathologies, mainly neuromuscular, sleep disorders, and chronic lung diseases, which require the continuous use of ventilatory support. ${ }^{2}$ Moreover, when faced with a respiratory infection, many among this particular group need advanced health care and special respiratory care to prevent hospitalization, such as increased ventilatory support, manual or mechanical assistance for cough, and/or respiratory physiotherapy at home. ${ }^{2}$

Many of these children receive various health care services through different home hospitalization programs, which depend on the severity of their diseases. In the current context, the visit to and monitoring of these 
patients should be limited to the essential services with adequate protection measures. In cases where professional visits are crucial, the homes must already have the protection measures recommended by the World Health Organization and the majority of health authorities around the world. ${ }^{3}$

In this group of patients, health care should be maintained through telemedicine systems to avoid any type of infection risk and not disrupt the medical and educational care of the children. However, we recommend providing the essential medical services and emergency professional visits whenever possible to maintain the minimum standard of health care. ${ }^{4}$

Despite these measures, these respiratory exacerbations usually have extended durations and higher probabilities of coinfection with other pathogens. ${ }^{5}$ For this reason, we need to prevent the increased risk of contagion and adding to the burden of the oversaturated health system; and if an infection occurs, we must act quickly to avoid respiratory deterioration in the patients because the prognosis of ICU-admitted patients is poor. ${ }^{1}$

In case a diagnosis of COVID-19 is confirmed in children with HMV, the professionals who deliver health care at home must have the necessary PPE (including N95/FFP2 mask or similar, goggles or face shield, long-sleeved water-resistant gown, and gloves). ${ }^{3}$ Caregivers must also have adequate protection measures for their care and accompaniment for as long as they are staying at home. In addition, home health care service must provide a contingency and protection plan for the caregivers in the event that the child user of invasive or non-invasive ventilation is COVID-19 positive. This plan must have the implements commonly used by health personnel in hospitals, an educational protocol for managing the patient, and telemedicine systems to facilitate monitoring.

At the time of respiratory infection, a possible assistance strategy for this group of patients is the modification of the ventilator parameters. ${ }^{2}$ If the patient uses non-invasive mechanical ventilation, then we must not forget that this intervention has been classified as a high-risk procedure for SARS-Cov-2 transmission because of the increased dispersion of droplets associated with the increased flow produced by the device. ${ }^{3}$ These droplets can even reach a distance of $92 \mathrm{~cm}$ from the expiratory port. ${ }^{3}$ In the period of acute infection, the delivery of inhaled drugs may be required. In this case, metered dose inhalers should be preferred, and if nebulization is unavoidable, the necessary measures must be taken to minimize the risk of droplet dispersion, such as the use of surgical masks over the cloud expelled by the nebulizer or the exit of caregivers from the patient's room during the procedure. ${ }^{3}$

Improving the effectiveness of respiratory care in children with HMV will achieve lower respiratory morbidity with subsequent lower utilization of health care resources, which is especially crucial in this time of collapsed health systems.

\section{CONFLICT OF INTERESTS}

The authors declare that there are no conflict of interests

\section{ORCID}

Luis Vasconcello-Castillo https://orcid.org/0000-0003-0405-3831

Rodrigo Torres-Castro https://orcid.org/0000-0001-7974-4333

Roberto Vera-Uribe https://orcid.org/0000-0003-4639-9574

\section{REFERENCES}

1. Jin Y, Yang H, Ji W, et al. Virology, Epidemiology, Pathogenesis, and Control of COVID-19. Viruses . 2020;12(4):372. doi:10.3390/v12040372

2. Amin R, MacLusky I, Zielinski D, et al. Pediatric home mechanical ventilation: A Canadian Thoracic Society clinical practice guideline executive summary. Can J Respir Crit Care, Sleep Med . 2017;1(1):7-36. doi: $10.1080 / 24745332.2017 .1300463$ 
3. Ferioli M, Cisternino C, Leo V, Pisani L, Palange P, Nava S. Protecting healthcare workers from SARSCoV-2 infection: practical indications. Eur Respir Rev . 2020;29(155). doi:10.1183/16000617.0068-2020

4. Veerapandiyan A, Wagner KR, Apkon S, et al. The care of patients with Duchenne, Becker and other muscular dystrophies in the COVID -19 pandemic . Muscle Nerve. April 2020. doi:10.1002/mus.26902

5. Buu MC. Respiratory complications, management and treatments for neuromuscular disease in children. Curr Opin Pediatr . 2017;29(3):326-333. doi:10.1097/MOP.0000000000000498 